\title{
SoxB2 in sea urchin development: implications in neurogenesis, ciliogenesis and skeletal patterning
}

\author{
Evgeniya Anishchenko, Maria Ina Arnone* and Salvatore D'Aniello* (1)
}

\begin{abstract}
Background: Current studies in evolutionary developmental biology are focused on the reconstruction of gene regulatory networks in target animal species. From decades, the scientific interest on genetic mechanisms orchestrating embryos development has been increasing in consequence to the fact that common features shared by evolutionarily distant phyla are being clarified. In 2011, a study across eumetazoan species showed for the first time the existence of a highly conserved non-coding element controlling the SoxB2 gene, which is involved in the early specification of the nervous system. This discovery raised several questions about SoxB2 function and regulation in deuterostomes from an evolutionary point of view.

Results: Due to the relevant phylogenetic position within deuterostomes, the sea urchin Strongylocentrotus purpuratus represents an advantageous animal model in the field of evolutionary developmental biology. Herein, we show a comprehensive study of SoxB2 functions in sea urchins, in particular its expression pattern in a wide range of developmental stages, and its co-localization with other neurogenic markers, as SoxB1, SoxC and Elav. Moreover, this work provides a detailed description of the phenotype of sea urchin SoxB2 knocked-down embryos, confirming its key function in neurogenesis and revealing, for the first time, its additional roles in oral and aboral ectoderm cilia and skeletal rod morphology.

Conclusions: We concluded that SOXB2 in sea urchins has a neurogenic function; however, this gene could have multiple roles in sea urchin embryogenesis, expanding its expression in non-neurogenic cells. We showed that SoxB2 is functionally conserved among deuterostomes and suggested that in S. purpuratus this gene acquired additional functions, being involved in ciliogenesis and skeletal patterning.
\end{abstract}

Keywords: Nervous system development, Ciliogenesis, Skeletal patterning, Evolution, Echinoderms

\section{Background}

Many studies have been undertaken in order to unravel the developmental significance of SoxB1 and SoxB2 crosstalk in the animal kingdom [1-4]. These genes are structurally very similar, but have antagonistic roles: So $x B 1$ is in fact considered a transcriptional activator, while SoxB2 a transcriptional repressor [5]. In all bilateria, they are mainly involved in development and cell specification [6], and despite the fact that they participate

*Correspondence: miarnone@szn.it; salvatore.daniello@szn.it Biology and Evolution of Marine Organisms, Stazione Zoologica Anton Dohrn Napoli, Villa Comunale, 80121 Naples, Italy to common processes, much attention has been paid to the role of SoxB1 in development, while the knowledge of SoxB2 functions is still quite limited. SoxB2 is known to be a neurogenic transcription factor (TF), and its importance has been re-evaluated by the finding of an ultra-conserved SoxB2 non-coding regulatory element discovered in distant metazoan phyla, from cnidarians to human [7].

The function of Sox21, the SoxB2 vertebrate's ortholog, has been mostly studied in fish, chicken and mouse. In chicken, it has a neurogenic function and it is expressed in vestibular and auditory organs, being an important regulator of sensory cell differentiation [8, 9]. In Xenopus 
laevis, Sox21 is expressed in several regions of the central nervous system (CNS) and in the sensory organs [4], similarly to chicken [10]. Moreover, Sox21 in zebrafish plays an important role in lens formation, embryonic CNS development, endoderm and ectoderm differentiation [11].

In vertebrates, it has been demonstrated the existence of functional redundancy among Sox family members $[12,13]$ and a major role in specification of several cell types and tissues seems to be due to their tendency to possess hypervariable cis-regulatory mechanisms. In invertebrate deuterostomes, SoxB2 has been studied in acorn worm, Ptychodera flava, in which it is expressed in the ciliary band, apical organ and foregut [14]. In the cephalochordate Branchiostoma belcheri, SoxB2b is expressed in the neural plate and subsequently in the neural tube and foregut [15].

In the present study, we focused our attention on SoxB2 during the development of the sea urchin S. purpuratus, belonging to echinoderms, which shares a common ancestor with modern chordates dating back about 500 million years [16]. Several studies were carried out in the purple sea urchin S. purpuratus at early developmental stages for maternally transmitted SoxB2 [1, 17-19]. In particular, Kenny and colleagues showed SoxB2 expression pattern and its putative function by knock-down experiments. They showed an involvement of SoxB2 into the oral ectoderm formation and body axes establishment during sea urchins gastrulation [1]. A more recent study demonstrated the implication of SoxB2 in neuronal specification up to $72 \mathrm{~h}$ post-fertilization (hpf) [19]. Nevertheless, there is still a lack of information regarding the SoxB2 expression pattern at later developmental stages, as well as a detailed study regarding SoxB2 nonneurogenic functions. Our study aimed to fill this gap in light of the recent comprehensive description of the sea urchin larval nervous system [20-24] showing for the first time the complete expression pattern including late developmental stages (144 hpf) and demonstrating its implication in multiple developmental processes, as NS specification, ciliogenesis and, intriguingly, skeletogenesis, the latter representing an echinoderm-specific ontogenetic mechanism.

\section{Results}

\section{Nervous system specification during sea urchin} development: orchestration by Sox genes expression While transcriptional data of SoxB1 and SoxB2 have been comprehensively included in the Echinoderms genome database (Echinobase) up to prism developmental stage, very little is known about late larval expression profile of these two genes. To fill this gap, we performed in situ hybridization experiments at blastula (24 hpf), early and late gastrula (30 and $48 \mathrm{hpf}$ ) and pluteus (72, 96, 120 and $144 \mathrm{hpf}$ ) stages, using both SoxB1 and SoxB2 riboprobes (Fig. 1). Moreover, although many studies addressed the interplay of these two genes in the developing nervous system $[1,3,4]$, their co-localization was never shown before. By using double fluorescent whole mount in situ hybridization (WISH) of SoxB1 and SoxB2, we analyzed their co-localization (Fig. 1 $\mathrm{a}^{\prime \prime}-\mathrm{h}^{\prime \prime}$ ). At blastula stage, the expression patterns of SoxB1 and SoxB2 overlap in the ectoderm (Fig. 1a", dashed line), with the exception of the vegetal pole. At early gastrula stage, SoxB1 is ubiquitous but mostly expressed in the animal and vegetal ectoderm (Fig. 1b, b"). SoxB2 has a similar expression pattern at this stage, except that it is absent in the apical ectoderm (Fig. 1b', dashed line). SoxB1 and SoxB2 are co-expressed in the dorsoventral and left-right ectoderm (Fig. $1 b^{\prime \prime}$, dashed line). At late gastrula stage, both genes are expressed mainly in the oral ectoderm (Fig. 1c- $\mathrm{c}^{\prime \prime}$, $\mathrm{d}-\mathrm{d}^{\prime \prime}$, dashed lines) and in the foregut (Fig. 1 $\mathrm{c}^{\prime \prime}$, arrow). At pluteus stage (72 hpf), both genes show strongest expression in the oral ectoderm around the ciliary band (Fig. 1e-e', $\mathrm{f}-\mathrm{f}^{\prime}$, dashed lines), and the aboral ectoderm lacks SoxB1 and SoxB2 expression. At $96 \mathrm{hpf}$, SoxB1 and $S o x B 2$ are still expressed within the ciliary band, but SoxB2 expression levels decrease in the apical organ (arrows in Fig. $\left.1 \mathrm{~g}^{\prime}-\mathrm{g}^{\prime \prime}\right)$. Later in development (120 hpf), SoxB1 partially disappears from tips of the oral arms (Fig. 1h, arrows) and SoxB2 remain unchanged in this area (Fig. $1 \mathrm{~h}^{\prime}$, arrows). At 144 hpf, SoxB1 and SoxB2 expression in ciliary band can be detected only using colorimetric WISH (Fig. 1i-i'), thanks to prolonged exposure of the enzymatic reaction. At this late larval stage, the expression of both genes is prevalent in oral and aboral arms of the embryo as shown in Fig. 1i-i' (arrows). Moreover, SoxB2 appear strongly expressed in a single cell at the pluteus apex (Fig. 1i', arrowhead).

Furthermore, to understand the crosstalk between SoxB2 and other genes implicated in neuronal differentiation, we carried out double fluorescent in situ hybridizations (FISH) in order to assess the co-localization of SoxB2 with neurogenic transcription factors, SoxC and Elav, which were previously described to have a sequential expression during sea urchins neurogenesis [25]. As Garner and colleagues recently showed by immunohistochemistry [19], we confirmed by FISH that some SoxC positive cells also express SoxB2 in 48 hpf embryos in the oral ectoderm (Fig. 2a, b). We highlighted novel expression domain for both genes in adjacent cells within the foregut, which only partially co-localize (Fig. 2a, b, arrows). On the other hand, we tested the expression of Elav that is a terminal marker of neuronal specification and demonstrated that it does not co-express with SoxB2 neither with $\operatorname{SoxC}$ (Fig. 2c, d). 

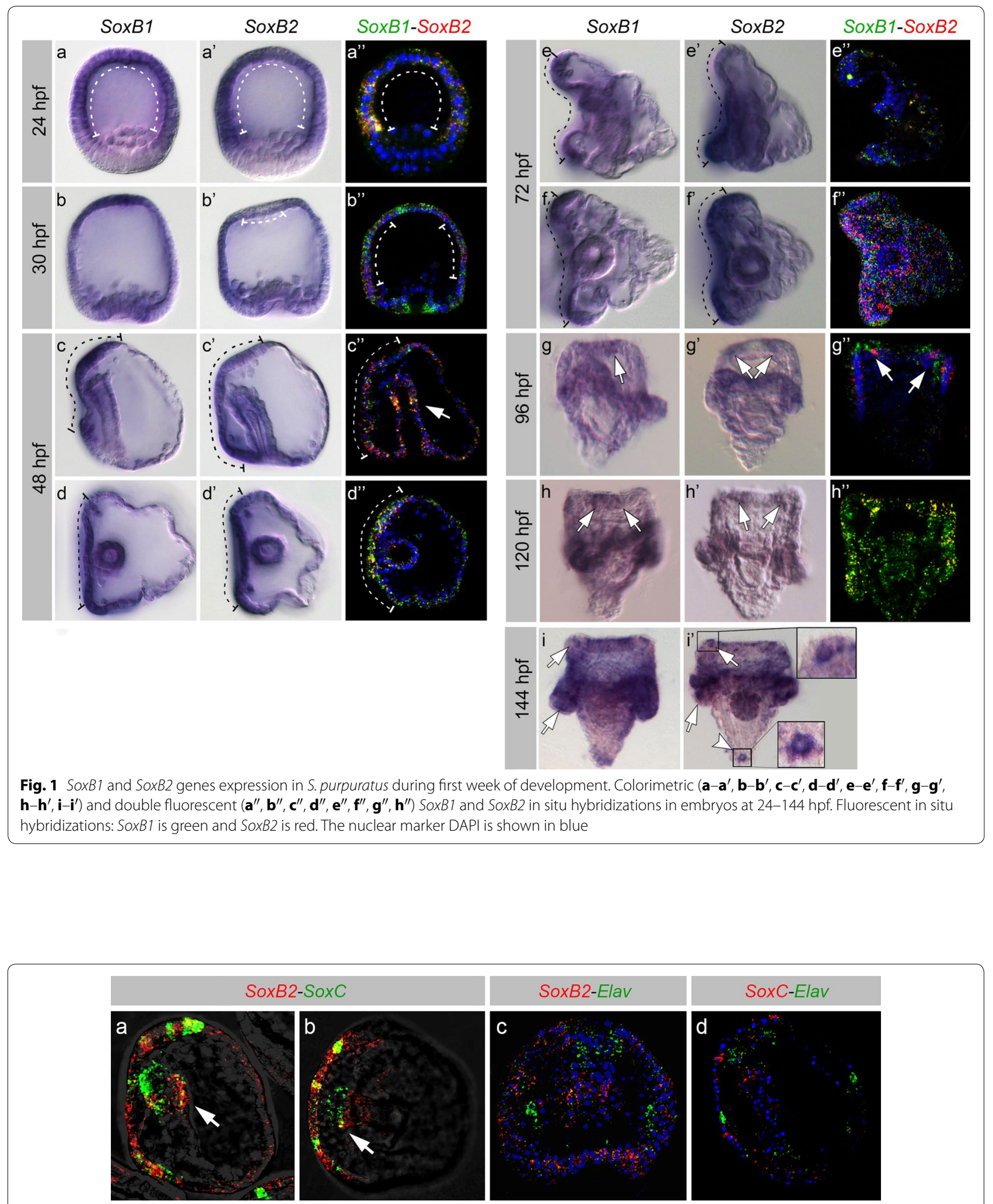

Fig. 2 SoxC, Elav and SoxB2 genes expression in S. purpuratus at 48 hpf. FISH in sea urchin embryos: SoxC is represented in green and SoxB2 in red. Embryos are shown from lateral (a) and apical (b) views. Regions of co-expression are shown in yellow. $\mathbf{c}$ FISH in sea urchin embryos; Elav is green and SoxB2 is red. Embryos are shown from the oral view. d FISH in sea urchin embryos; Elav is green and SoxC is red. Embryos are shown from lateral view. DAPI is shown in blue 


\section{SoxB2 functional and molecular characterization}

Previous studies showed that SoxB2 knock-down led to body axes alterations and gastrulation arrest [1]. Herein, we used identical morpholino antisense oligonucleotides (MO) sequences that, injected at a lower concentration compared to what reported in [1], led to a less severe phenotype, allowing gastrulation to occur and revealing other developmental defects. The analysis of the obtained phenotype permitted to describe additional developmental processes that require SoxB2 activity. We monitored the general morphology and nervous system development of SoxB2 morphant embryos during the first $72 \mathrm{hpf}$. The most evident phenotype in 48 and 72 hpf SoxB2 morphants was a prevalence of embryos with roundish-shaped body showing defects in the characteristic oral ectoderm (Fig. 3b-c $\mathrm{c}^{\prime \prime}$, e- $\mathrm{f}^{\prime \prime}$ ). Noteworthy, at $48 \mathrm{hpf}$ SoxB2-MOs affect dorsoventral connecting rods (DVC) and body rods (BR) which are shorter than in controls (Fig. $3 \mathrm{~b}^{\prime}, \mathrm{b}^{\prime \prime}$; arrowhead and arrows, respectively). Body rods appear slightly curved (Fig. 3c" arrow), while ventral transverse rods (VT) in MO-1 and MO-2 embryos remain similar to those in controls (Fig. $3 \mathrm{a}^{\prime \prime}-\mathrm{c}^{\prime \prime}$, arrows). Later in development, at $72 \mathrm{hpf}$, several defects have been observed such as additional ramification of BRs (Fig. 3e'), outgrowth of posterior anal rods (AR) (Fig. 3f') and abnormal growth of the BRs that do not converge in the apex as it normally occurs (Fig. 3e', $\mathrm{f}^{\prime \prime}$, arrows) [26, 27].

We injected two different SoxB2 morpholino antisense oligonucleotides: MO-1 (identical to the one used by Kenny and colleagues [1] and MO-2, which evoked similar phenotypes in at least $97 \%$ of injected embryos (Fig. 3b-b", e-e $\mathrm{e}^{\prime \prime}, \mathrm{c}-\mathrm{c}^{\prime \prime}, \mathrm{f}-\mathrm{f}^{\prime \prime}$, respectively). The efficiency of our microinjection experiments and the specificity of $\mathrm{MO}$ effects were controlled using a fluorescein-tagged standard control MO (MO-Fluo) that showed green fluorescent signal in $95 \%$ injected embryos. The morphology of sea urchin embryos injected with MO-Fluo was comparable to the uninjected controls, and no skeletal defects were ever observed in such control injection performed side by side with SoxB2 MO-1 and MO-2 injection on the same batch of embryos (Additional file 1: Figure S1). The survival rate of all injected embryos was $>90 \%$.

The detailed analysis of NS and ciliary band formation in SoxB2 morphants was performed in embryos at $72 \mathrm{hpf}$ by immunohistochemical analysis (IHC) using antiacetylated tubulin (AcTub) to stain ciliated structures, anti-serotonin (Ser) and anti-synaptotagmin B (1e11) to detect neuronal networks. NS alterations were observed in SoxB2 morphants, in fact $72 \mathrm{hpf}$ sea urchin embryos injected with SoxB2 MO-1 showed that the number of synaptotagmin $B$ positive cells was significantly decreased (> 50\%), mostly at the expense of ganglial cells (Fig. 4b-b", arrows; and Additional file 2: Figure S2a) compared to uninjected controls (Fig. 4a- $\mathrm{a}^{\prime \prime}$ ) and to MOFluo injected embryos (Additional file 3: Figure S4d). As shown in Fig. 4b, the nerve ring, associated with the ciliary band and normally encircling the border of the oral ectoderm, appeared incomplete in pluteus stage (72 hpf) morphants, when compared with controls (Fig. 4a). Moreover, many neuronal cells lost axonal connections. The neuronal circle around the mouth was altered in morphants (Fig. 4b, arrows), and neurites did not project toward the posterior end of the larval body, compared to the control embryos (Fig. 4a', b'). The number of serotonergic neurons decreased of $40 \%$ in the sea urchin SoxB2 morphant larvae (Fig. 4b, arrowhead; Additional file 2: Figure S2b). This phenotype was detected in $85 \%$ of the injected embryos in six biological replicates.

Furthermore, our analysis showed that the morphants presented significantly longer cilia $(21 \pm 2 \mu \mathrm{m})$, compared to uninjected controls $(11 \pm 1 \mu \mathrm{m})$ (Fig. $4 \mathrm{c}-\mathrm{d}^{\prime \prime}$; Additional file 2: Figure S2c). Interestingly, longer cilia were observed even outside the innervated ciliary band over all the embryos body (Fig. $4 \mathrm{~d}^{\prime}-\mathrm{d}^{\prime \prime}$, arrows). The observed differences in cilia length are shown in Additional file 2: Figure S2c and Additional file 4: Figure S3. Control embryos, injected with $200 \mu \mathrm{M}$ MO-Fluo, did not show significantly longer cilia compared to uninjected controls, confirming the specificity of SoxB2 knock-down phenotype (Additional file 4: Figure S3d).

In order to enrich our knowledge on SoxB2 function in sea urchin embryogenesis and to propose its possible molecular mechanisms, we integrated our study including expression levels of genes fundamental for ectoderm, nervous system, ciliary band formation, and skeletogenesis. To this aim, we performed qPCR analysis on mRNA extracted from 48 and 72 hpf SoxB2 morphant embryos, compared with uninjected controls, to study the transcriptional alteration of selected ectodermal genes: Onecut, SoxB1, SoxC, Pax2/5/8; mesodermal genes: Vegf, Bmp3, SM30, SM50; and an ecto-endodermal gene: Brn1/2/4.

Among all neurogenic genes taking part in ciliary band formation Onecut is the only one that appeared significantly decreased in SoxB2 morphants according to qPCR (Fig. 5a) and WISH experiments (Fig. 5b, c). Vegf, a gene known for its key role in sea urchin larval skeleton formation [26] resulted downregulated, while SM50, $\mathrm{Pax} 2 / 5 / 8$ and Bmp3 showed a strong upregulation (Fig. 5a, d).

\section{Discussion}

In the present work, we demonstrated that in sea urchin S. purpuratus embryos SoxB2 and its paralog SoxB1 co-localize at different developmental stages. We also showed their expression patterns at late developmental stages (96-144 hpf) and SoxB2 co-localization with SoxC 


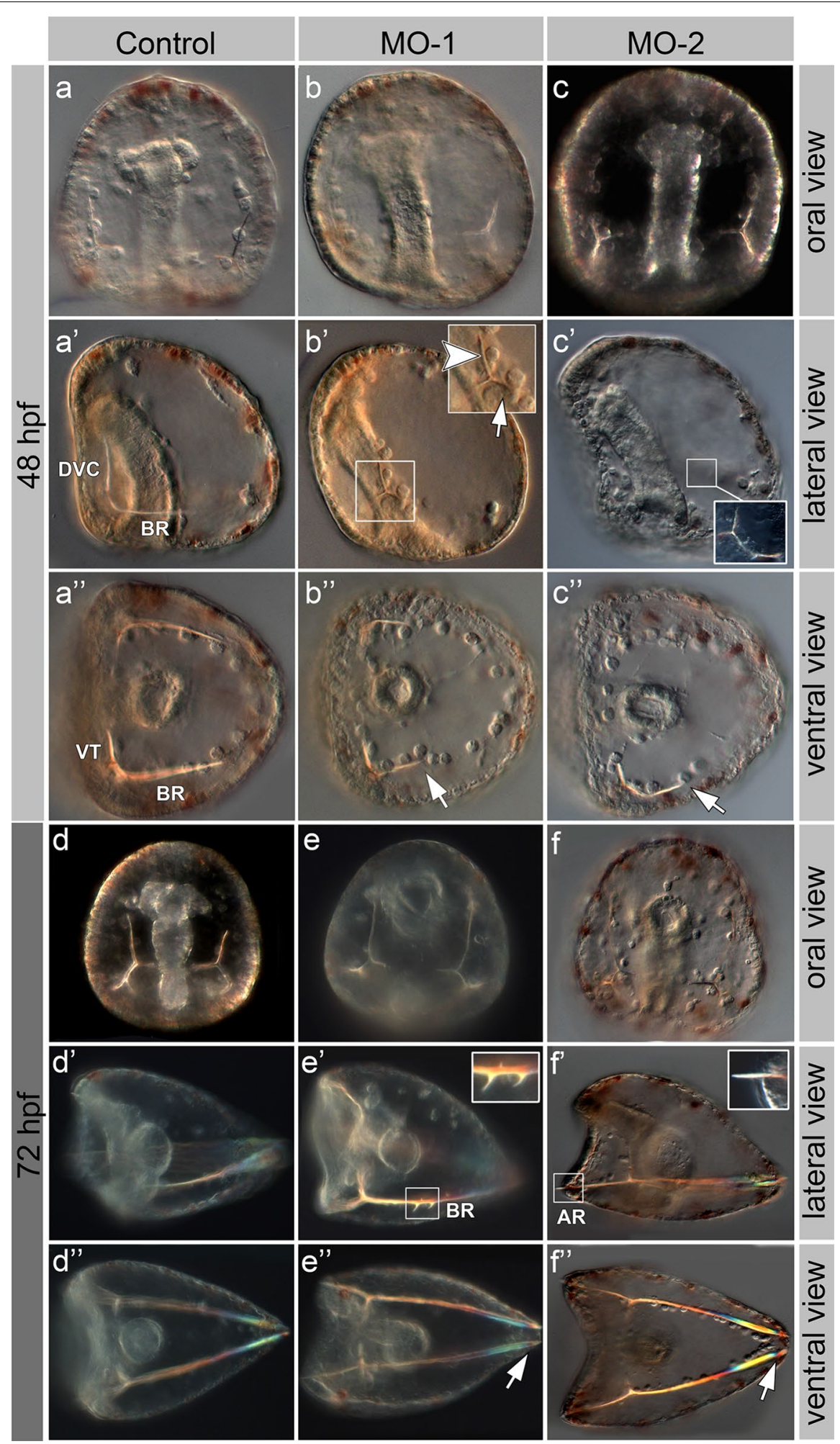

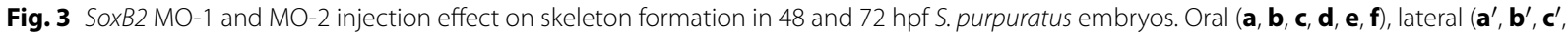
$\left.\mathbf{d}^{\prime}, \mathbf{e}^{\prime}, \mathbf{f}^{\prime}\right)$ and vegetal $\left(\mathbf{a}^{\prime \prime}, \mathbf{b}^{\prime \prime}, \mathbf{c}^{\prime \prime}, \mathbf{d}^{\prime \prime}, \mathbf{e}^{\prime \prime}, \mathbf{f}^{\prime \prime}\right)$ view of embryos at $48 \mathrm{hpf}\left(\mathbf{a}-\mathbf{c}^{\prime \prime}\right)$ and $72 \mathrm{hpf}\left(\mathbf{d}-\mathbf{f}^{\prime \prime}\right)$. Uninjected control embryos $\left(\mathbf{a}-\mathbf{a}^{\prime \prime}\right.$ and $\left.\mathbf{d}-\mathbf{d}^{\prime \prime}\right)$ were compared with morphants, injected with SoxB2 MO-1 (b-b", $\left.\mathbf{e}^{\prime \prime}-\mathbf{e}^{\prime \prime}\right)$ and SoxB2 MO-2 (c-c' $\left.\mathbf{c}^{\prime \prime}, \mathbf{f}-\mathbf{f}^{\prime \prime}\right)$ 

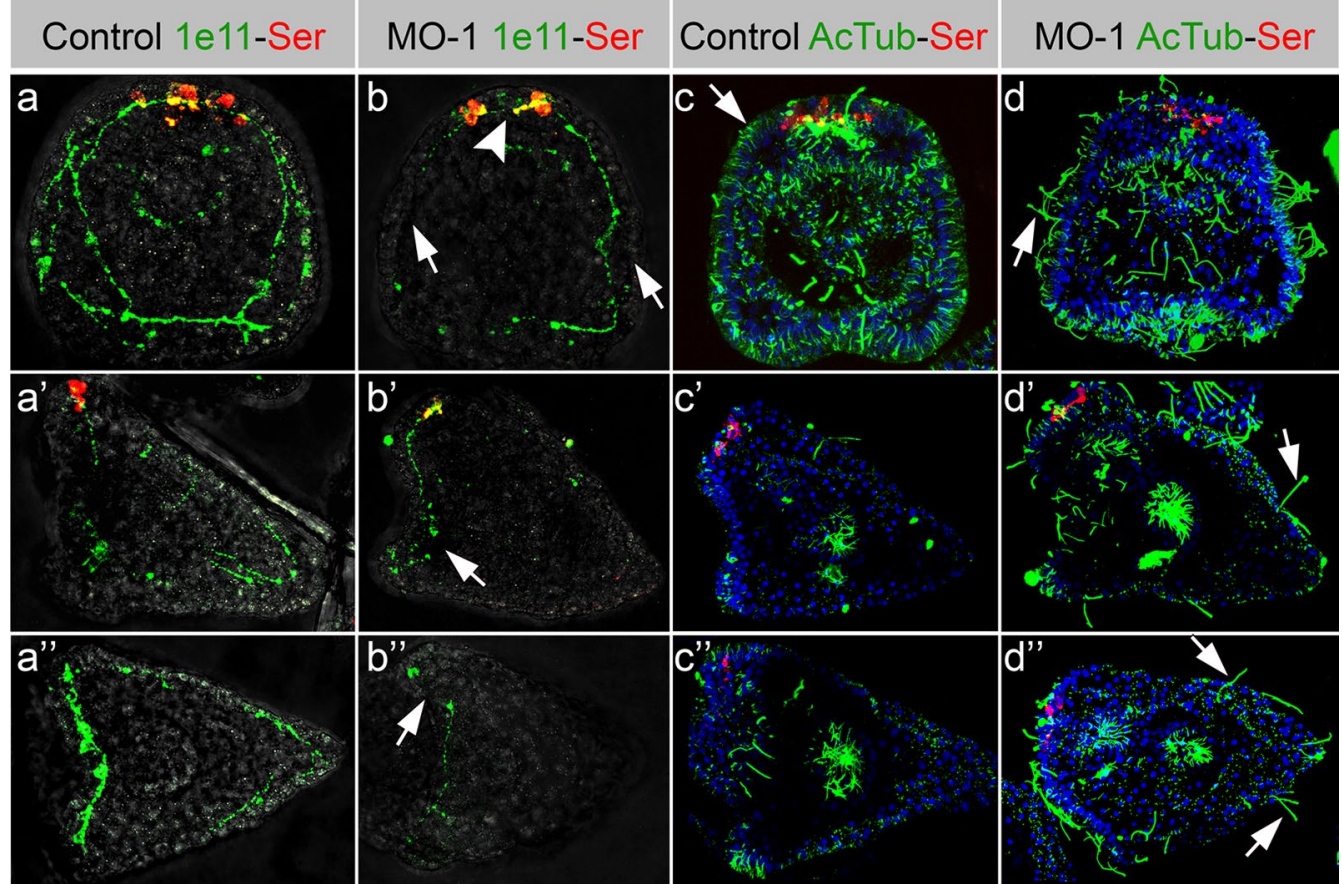

Fig. 4 SoxB2 MO-1 injection effect on nervous system formation and ciliogenesis in 72 hpf S. purpuratus embryos. Oral (a, b, c, d), lateral (a', $\mathbf{b}^{\prime}, \mathbf{c}^{\prime}$, $\mathbf{d}^{\prime}$ ) and vegetal $\left(\mathbf{a}^{\prime \prime}, \mathbf{b}^{\prime \prime}, \mathbf{c}^{\prime \prime}, \mathbf{d}^{\prime \prime}\right)$ views of embryos at $72 \mathrm{hpf}$. Uninjected control embryos (a-a' $\mathbf{a}^{\prime \prime}$ and $\left.\mathbf{c}-\mathbf{c}^{\prime \prime}\right)$ were compared with morphants, injected with SoxB2 MO-1 (b-b"), $\left.\mathbf{d}-\mathbf{d}^{\prime \prime}\right)$. Serotonin is shown in red, DAPI in blue, Synaptotagmin B (1e11) in $\mathbf{a}-\mathbf{b}^{\prime \prime}$ and AcTubulin in c- $\mathbf{d}^{\prime \prime}$ are shown in green

and Elav. SoxB2 and SoxB1 temporal and spatial expression patterns are quite similar at blastula and gastrula stages, and they co-occurred in the ectoderm at blastula stage and additionally appeared in foregut cells at gastrula stage. These data confirm previously known expression patterns of SoxB1 and SoxB2 [1, 2], even though the co-expression of these two genes had not been shown before. The main differences in their embryonic localization become clear at $30 \mathrm{hpf}$, when the vegetal pole and the animal pole domains (APD) [25, 27] express SoxB1, while APD lacks SoxB2, in agreement with what already shown by Garner et al. [19] at $48 \mathrm{hpf}$. At this developmental stage, SoxB2 appears expressed (and co-localized with SoxB1) in oral ectoderm and in foregut, which has endodermal origin. Therefore, the expression of SoxB2 in sea urchins is not limited to ectodermal tissues. This appears as an evolutionarily conserved feature, since endodermal expression of this gene has been already observed in chordates (amphioxus) [15]. Sea urchin foregut neurons are known to differentiate in loco; therefore, they do not derive from ectodermal precursors neither from migrating APD cells [18]. We here speculate that SoxB2 could be a potential regulator of neurogenesis in the foregut. This hypothesis is reinforced by double WISH experiments demonstrating that SoxB2 and the late nervous system marker, $\operatorname{Sox} C$, are expressed in different cells within the foregut (Fig. 2a, b, arrow), reflecting an intermediate state of progression of foregut neurons maturation, with SoxB2 playing a role in its initial phase. APD cells, in fact, express Elav that is a terminal factor of neuronal specification [19], and do not express SoxB2 or SoxC. Hence, these data could have an impact in our understanding of the timing and hierarchy of foregut innervation.

The key role of SoxB2 in neurogenesis was confirmed by the MO knock-down at $72 \mathrm{hpf}$ that showed developmental abnormalities in oral neuronal ring with incomplete axonal connections and in ciliated structures that resulted longer than control embryos and, unlike controls, distributed along the whole body. These alterations were confirmed by qPCR analysis in which the ciliary band specification gene Onecut appeared as the only gene altered among proneural ectodermal genes tested in our experiments, while SoxB1, SoxC and Brn1/2/4 remained unaffected. WISH experiments showed that Onecut expression domain was significantly reduced (Fig. 5).

In addition, we characterized the SoxB2 expression pattern at late developmental stages, $144 \mathrm{hpf}$ pluteus stage, in which the NS is already formed and SoxB2 acquires a new expression domain in the apex, where important morphogenetic processes shaping the skeleton occur at late pluteus stage. Therefore, SoxB2 expression profile in $S$. purpuratus embryos suggests an alternative function 


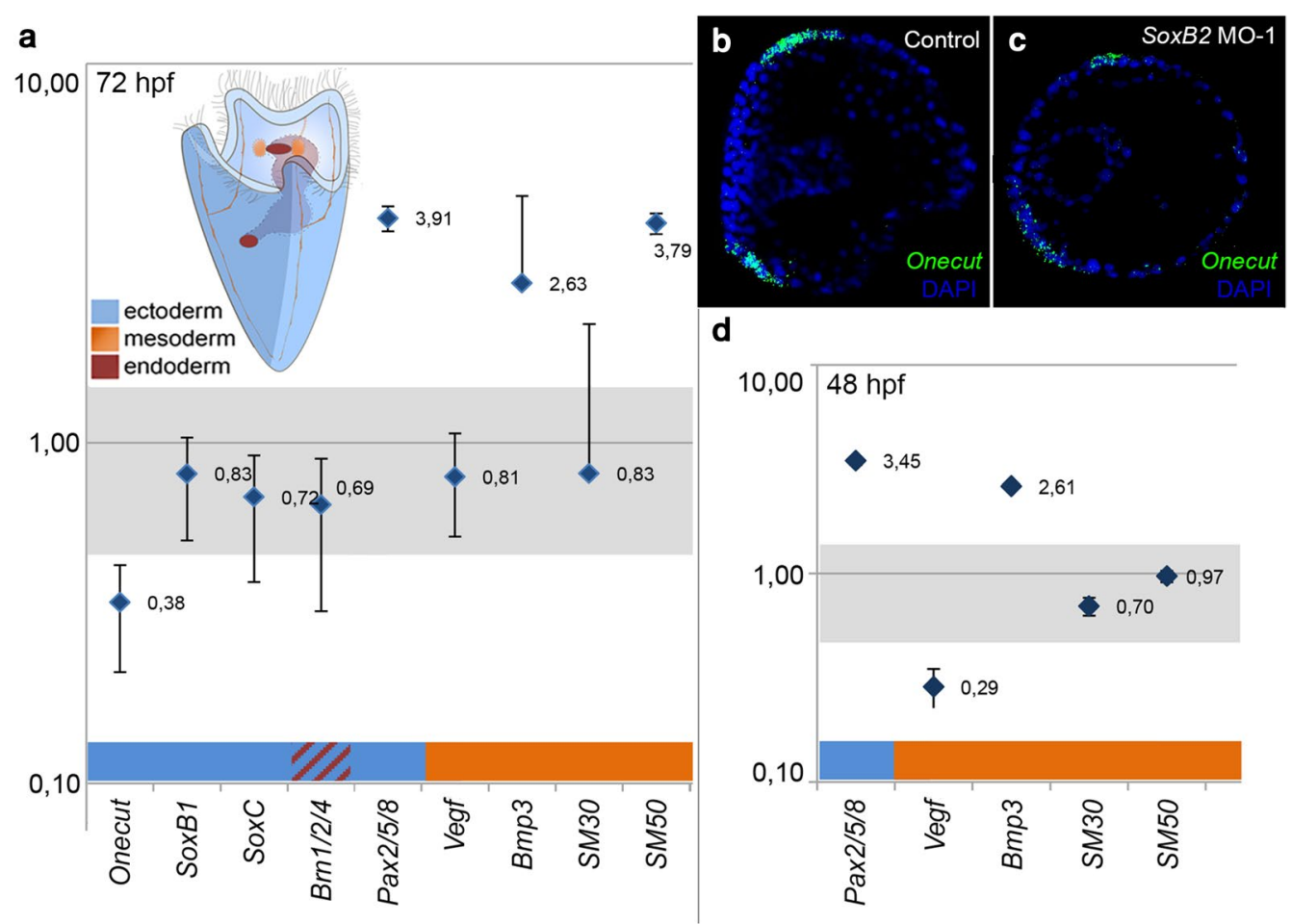

Fig. 5 Expression levels of developmental genes in S. purpuratus SoxB2 morphants. Ratio of gene expression levels comparing SoxB2 morphants and uninjected control embryos at $72 \mathrm{hpf}(\mathbf{a})$ and $48 \mathrm{hpf}(\mathbf{d})$ by qPCR. Significant alterations in gene expression are below and above the gray bar (value \pm 0.5). The histograms are represented in a Log10 scale for the $y$-axis. Decreasing levels of SoxB1 (green) and Onecut (red) expression in SoxB2 morphants was shown using FISH (c). Morphants were compared with uninjected control sea urchin embryos at $48 \mathrm{hpf}$, the images were taken

from the apical view $(\mathbf{b})$

to neurogenesis, suggesting implication in endoskeleton patterning. This hypothesis is further supported by the SoxB2 knock-down phenotype. In fact, SoxB2 morphants showed the reduction of spicules at $48 \mathrm{hpf}$ and later the ramification and extension of the ventral spicules over the body at $72 \mathrm{hpf}$ accompanied by alterations in their orientation and failure to convergence at the apex. The observed overexpression of Vegf, Bmp3 and SM5O (sea urchin embryo spicules) in SoxB2 morphants positively correlates with the aberration of the endoskeleton structure (Fig. 5). In fact, $P a x 2 / 5 / 8$, known to control primary mesenchyme cells specification and skeletogenesis via Vegf, Bmp3, SM30 and SM50 [28], resulted overexpressed in SoxB2 morphants.

Many efforts have been dedicated in sea urchin developmental biology to understand individually the formation and regulation of skeletal patterning [28] as well as oral ectoderm specification [20, 25, 29] and differentiation of the innervated ciliated band [19, 24]. Based on experimental evidences reported in the present work, we hypothesized that SoxB2 could be the cross-linking GRN factor that orchestrates the developmental and functional organization of derivatives from all three embryonic layers in sea urchin embryos: nervous system (ectoderm), foregut (endoderm) and endoskeleton (mesoderm). Nevertheless, in other animals, SoxB2 is prevalently a key regulator of the nervous system specification, as shown in cnidarian Nematostella vectensis [30,31], in fruit fly Drosophila melanogaster [7], in hemichordate Ptychodera flava [14] and in chordates amphioxus [15, $32]$ and vertebrates $[5,6,9,10]$. The only other example of non-neuronal roles so far described in other metazoans is represented by the amphioxus SoxB2 that has been shown to be involved in the embryonic gut $[15,32]$. Therefore, it can be speculated that the SoxB2 gene has been co-opted in sea urchin skeletogenesis.

\section{Conclusions}

SoxB2 in metazoans has a broad function in orchestrating NS at early stages of development. We here show that, at certain developmental stages, SoxB2 in sea urchin embryos acquires secondary regulatory roles in foregut innervation, skeletogenesis and ciliogenesis. Moreover, the example of SoxB2 role in sea urchin skeletogenesis bring to the discussion the Evo-Devo concept that novelty in evolution can also rise from ultra-conserved and 
stereotyped gene pathways without drastic genomic rearrangements. Interestingly, within echinoderms, the embryonic skeletogenesis is a lineage-specific process exclusively observed in Echinoids and Ophiuroids [33, 34]. Therefore, it would be interesting, in the future, to further investigate the putative co-option event that allowed SoxB2 to acquire exclusive features in echinoderms.

\section{Methods}

\section{Animal husbandry and embryo culture}

Adult S. purpuratus were provided by Patrick Leahy (Kerchoff Marine Laboratory, California Institute of Technology, Pasadena, CA, USA) and housed in circulating seawater aquaria at $16{ }^{\circ} \mathrm{C}$ at the Stazione Zoologica Anton Dohrn, Napoli (Italy). Gamete release was induced by vigorously shaking ripe animals. The embryos were cultured in $0.22 \mu \mathrm{m}$ filtered Mediterranean sea water, diluted with deionized $\mathrm{H}_{2} \mathrm{O}$ in a 9:1 ratio.

\section{Whole mount in situ hybridization}

SoxB1 (AF157389; SPU_022820), SoxB2 (ABI53357.1; SPU_025113) and SoxC (NP_001158501; SPU_002603) cDNA were obtained by RT-PCR on total RNA that was isolated from 72 hpf $S$. purpuratus embryos using RNAqueous-Micro Kit (Ambion). The retro-transcription was carried out with the VILO SuperScript cDNA Synthesis kit (Invitrogen). cDNA amplification was performed using following primers: SoxB1-F: $5^{\prime}$-GTC TGT TCC TGG TGT ACA TG-3'; SoxB1-R: 5'-TAC ATA TGC GTG AGT GGA AC-3'; SoxB2-F: $5^{\prime}$-ATG ATG ATG GAC TCT GCG ATG G-3'; SoxB2-R: $5^{\prime}$-AGA GAG CCG TCG CCG CTG TCG G-3'; SoxC-F: 5'-GTT CCT CAG AAG AGC TTC GC-3'; SoxC-R: 5'-AGC AAT CGT CCA TGT CGA C-3'. cDNA fragments were cloned into p-GEM-T Easy vector (Promega). Onecut riboprobe was prepared using the sequence published by Poustka et al. [29]. Elav riboprobe was synthesized using a clonal plasmid provided by Dr. Paola Oliveri (University College London, UK; unpublished data). Gene clones were used for RNA probe synthesis with DNaseI-RNAse (Roche) and labeled with Digoxigenin or Fluorescein (Roche), according to the supplier's protocol. For single colorimetric in situ hybridizations, we followed the protocol as was previously described [35] with following modifications: hybridization with RNA probe was performed at $63{ }^{\circ} \mathrm{C}$ overnight in $50 \%$ Formamide hybridization buffer. Double fluorescent in situ hybridization was performed as described in Cole et al. [36]. For differential interference contrast images, a Zeiss Axio Imager M1 microscope equipped with an "Axiocam" digital camera was used. Fluorescence images were obtained using a Zeiss confocal laser scanning LSM 510 microscope.

\section{Immunohistochemistry}

$72 \mathrm{hpf}$ embryos were fixed and used for immunohistochemistry procedure as outlined in Burke et al. [21]. In order to localize acetylated tubulin, serotonergic and synaptotagmin B positive cells we used 1:400 diluted mouse monoclonal anti-acetylated tubulin antibody (AcTub; T7451, Sigma-Aldrich), rabbit monoclonal anti-serotonin antibody (Ser; S55451E11, Sigma-Aldrich) and 1:50 diluted mouse monoclonal anti-synaptotagmin B antibody (1e11) [21], respectively. The fluorescent staining was developed using goat anti-Mouse IgG Alexa Fluor ${ }^{\circledR} 488$ conjugate (A-110010, Invitrogen) and Donkey anti-Rabbit Alexa Fluor $^{\circledR} 555$ conjugate (A-31572, Invitrogen) (1:500) secondary antibodies. Lastly, the embryos were incubated with nuclear marker DAPI (Sigma-Aldrich) 1:10000 in PBT (phosphate-buffered saline; 0.1\% Tween 20).

\section{Knock-down by morpholino antisense oligonucleotides microinjections}

Morpholino antisense oligonucleotides against SoxB2 translation were designed and acquired from Gene Tools: MO-1: 5'-TCC CCA TCG CAG AGT CCA TCA TCA T-3'; MO-2 5'-GTC GGA TGC TGG CTT TCA AAA CAG A-3'). MOs were injected in approximately 500 embryos in quantity of $2 \mathrm{pl}$ in each embryo. Injected solution contained $200 \mu \mathrm{M} \mathrm{MO}$ and $0.12 \mathrm{M} \mathrm{KCl}$. Fluorescein-tagged standard control MO (MO-Fluo: 5'-CCT CTT ACC TCA GTT ACA ATT TAT A-3') was used as a control and injected at $200 \mu \mathrm{M}$ concentration in 250 embryos in all experiments. Embryos injected with MO-Fluo showed bright fluorescence; their phenotype corresponds to the phenotype of uninjected embryos at 20-96 hpf. Experiments were repeated at least six times. The survival rate of $200 \mu \mathrm{M} \mathrm{MO}-1$ and MO-2 injected embryos was $>90 \%$ in all experiments. The morphant phenotype was observed in $>97 \%$ of injected survived embryos.

\section{Quantitative PCR}

The qPCR was carried out in a ViiATM 7 Real-Time PCR System (Applied Biosystems) using the SYBR ${ }^{\mathrm{TM}}$ Green reagent (Life Technologies). The expression levels of several sea urchin developmental genes that could be affected by SoxB2 (Onecut, SoxB1, SoxC, Brn1/2/4, Pax 2/5/8, Vegf, Bmp3, SM30 and SM50) were explored by $\mathrm{qPCR}$ using cDNA derived from 300 morphant embryos at 48 and $72 \mathrm{hpf}$ injected with $200 \mu \mathrm{M}$ SoxB2 MO-1. cDNA was synthesized using RNAqueous-Micro Kit (Ambion). Ubiquitin and Ef1a were used for data normalization [37, 38]. Primer sequences used in the qPCR analyses are listed in Additional file 5: Table S1. Gene expression levels of the morphants were compared with those of uninjected embryos. Each qPCR experiment was 
performed on three independent biological replicas, and each reaction was repeated three times.

\section{Additional files}

Additional file 1: Figure S1. Control MO-Fluo in $72 \mathrm{hpf} \mathrm{S.} \mathrm{purpuratus}$ embryos. Sea urchin embryos at $72 \mathrm{hpf}$ from oral $(\mathbf{a}, \mathbf{b}, \mathbf{c})$, lateral $\left(\mathbf{a}^{\prime}, \mathbf{b}^{\prime}, \mathbf{c}^{\prime}\right)$ and vegetal $\left(\mathbf{a}^{\prime \prime}, \mathbf{b}^{\prime \prime}, \mathbf{c}^{\prime \prime}\right)$ views depict the tissues where fluorescence deriving from the fluorescent $\mathrm{MO}$ is visible $\left(\mathbf{c}-\mathbf{c}^{\prime \prime}\right)$. Morphant embryos imaged with microscope $\left(\mathbf{b}-\mathbf{b}^{\prime \prime}\right)$ present a phenotype similar to uninjected control embryos (a-a').

Additional file 2: Figure S2. Statistical analysis of serotonergic neurons number and cilia length in $72 \mathrm{hpf}$ S. purpuratus SoxB2 knock-down experiments. a Embryonic nervous system (Synaptotagmin B by $1 \mathrm{e} 11$ immunohistochemistry) of uninjected control, MO-1, MO-2 and MO-Fluo embryos. The fluorescence of $1 \mathrm{e} 11$ positive neurons is shown in \%, normalized by control uninjected embryos (100\%). The intensity of staining from 10 embryos of each group was measured using ImageJ in three independent experiments. b Number of serotonergic neurons observed in six independent experiments using uninjected control, MO-1, MO-2 and MO-Fluo embryos. Serotonergic positive neurons were measured from at least 33 embryos in each experimental group. c Cilia length in uninjected control, MO-1, MO-2 and MO-Fluo embryos measured using the Zeiss confocal laser scanning LSM 510 microscope software. 10-12 cilia from at least 33 embryos were used in three independent experiments. Statistical analysis was performed using Prism 5 GraphPad software: $P$ value versus uninjected controls $={ }^{*} P<0.05$, ${ }^{* *} P<0.01$, ${ }^{* * *} P<0.001$, while $P$ value versus MO-Fluo $={ }^{+} P<0.05,{ }^{++} p<0.01,{ }^{+++} p<0.001$.

Additional file 3: Figure S4. Injection of MO-Fluo did not affect the development of Synaptotagmin B expressing neurons.

Additional file 4: Figure S3. Analysis of the cilia length in MO-injected embryos performed at $72 \mathrm{hpf}$ pluteus. AcTubulin staining is shown in green and DAPI in blue. a control non injected embryo, b MO-1 injected embryo, c MO-2 injected embryo, d MO-Fluo injected embryo. Measurements of longest cilia length (I) are indicated in white. All cilia length measurements were performed using Ziess LSM Image Browser software. 10-12 cilia from at least 33 embryos in every experimental group were measured; scale bar is $20 \mu \mathrm{m}$.

Additional file 5: Table S1. List of oligonucleotides used for qPCR experiments.

\section{Abbreviations}

AcTub: acetylated tubulin; bp: base pairs; DAPI: 4',6-diamidino-2-phenylindole; CNS: central nervous system; DIG: digoxigenin; FISH: fluorescent in situ hybridization; MO: morpholino antisense oligonucleotide; MO-Fluo: fluorescein-tagged standard control MO; hpf: hours post-fertilization; IHC: immunohistochemistry; NS: nervous system; qPCR: quantitative polymerase chain reaction; Ser: serotonin; TF: transcription factor; WISH: whole mount in situ hybridization.

\section{Authors' contributions}

EA carried out animals spawning and embryos fixation, molecular cloning, in situ hybridizations, immunohistochemistry, microscopic and confocal imaging, $\mathrm{QPCR}$, data analysis, prepared the figures and wrote the manuscript. MIA performed $\mathrm{MO}$ microinjections, contributed to the design of the study and to the data analysis. SDA conceived the study, contributed to the design of the study and to the data analysis, wrote the manuscript. All authors read and approved the final manuscript.

\section{Acknowledgements}

The authors are grateful to MARER of the Stazione Zoologica Anton Dohrn di Napoli for assistance with living organisms. Heartfelt gratitude to Dr. Yi-Hsien Su, Academia Sinica of Taipei (Taiwan), for her useful suggestions. We would like to acknowledge Dr. Paola Oliveri (UCL, UK) and Dr. Robert D. Burke (STN CSC, Victoria, Canada) for providing Elav clone and 1e11 antibody, respectively.
Competing interests

The authors declare that they have no competing interests.

Availability of data and materials

All data generated or analyzed during this study are included in this published article.

\section{Consent for publication}

Not applicable.

\section{Ethics approval and consent to participate}

No human subjects were used in this study, and the invertebrate animals used in this study are not subject to regulation by animal ethics committees.

\section{Funding}

Evgeniya Anishchenko was supported by a SZN PhD fellowship (2011-2014).

\section{Publisher's Note}

Springer Nature remains neutral with regard to jurisdictional claims in published maps and institutional affiliations.

Received: 26 October 2017 Accepted: 1 February 2018

Published online: 19 February 2018

\section{References}

1. Kenny AP, Oleksyn DW, Newman LA, Angerer RC, Angerer LM. Tight regulation of SpSoxB factors is required for patterning and morphogenesis in sea urchin embryos. Dev Biol. 2003;261:412-25.

2. Kenny AP, Kozlowski D, Oleksyn DW, Angerer LM, Angerer RC. SpSoxB1, a maternally encoded transcription factor asymmetrically distributed among early sea urchin blastomeres. Development. 1999;126:5473-83.

3. Uchikawa M, Yoshida M, Iwafuchi-Doi M, Matsuda K, Ishida Y, Takemoto T, et al. B1 and B2 Sox gene expression during neural plate development in chicken and mouse embryos: universal versus species-dependent features. Dev Growth Differ. 2011;53:761-71.

4. Cunningham DD, Meng Z, Fritzsch B, Casey ES. Cloning and developmental expression of the soxB2 genes, sox 14 and sox21, during Xenopus laevis embryogenesis. Int J Dev Biol. 2008;52:999-1004.

5. Kamachi Y, Kondoh $\mathrm{H}$. Sox proteins: regulators of cell fate specification and differentiation. Development. 2013;140:4129-44.

6. Kondoh $\mathrm{H}$, Kamachi Y. SOX-partner code for cell specification: regulatory target selection and underlying molecular mechanisms. Int J Biochem Cell Biol. 2010:42:391-9.

7. Royo JL, Maeso I, Irimia M, Gao F, Peter IS, Lopes CS, et al. Transphyletic conservation of developmental regulatory state in animal evolution. Proc Natl Acad Sci USA. 2011;108:14186-91.

8. Freeman SD, Daudet N. Artificial induction of Sox21 regulates sensory cell formation in the embryonic chicken inner ear. PLoS ONE. 2012;7:e46387.

9. Sandberg M, Källström M, Muhr J. Sox21 promotes the progression of vertebrate neurogenesis. Nat Neurosci. 2005;8:995-1001.

10. Uchikawa M, Kamachi Y, Kondoh H. Two distinct subgroups of Group B Sox genes for transcriptional activators and repressors: their expression during embryonic organogenesis of the chicken. Mech Dev. 1999:84:103-20.

11. Pauls S, Smith SF, Elgar G. Lens development depends on a pair of highly conserved Sox21 regulatory elements. Dev Biol. 2012;365:310-8.

12. Okuda Y, Yoda H, Uchikawa M, Furutani-Seiki M, Takeda H, Kondoh H, et al. Comparative genomic and expression analysis of group B1 sox genes in zebrafish indicates their diversification during vertebrate evolution. Dev Dyn. 2006;235:811-25.

13. Kamachi Y, Iwafuchi M, Okuda Y, Takemoto T, Uchikawa M, Kondoh H. Evolution of non-coding regulatory sequences involved in the developmental process: reflection of differential employment of paralogous genes as highlighted by Sox2 and group B1 Sox genes. Proc Jpn Acad Ser B Phys Biol Sci. 2009;85:55-68.

14. Taguchi S, Tagawa K, Humphreys T, Satoh N. Group BSoxGenes that contribute to specification of the vertebrate brain are expressed in the apical organ and ciliary bands of hemichordate larvae. Zool Sci. 2002;19:57-66. 
15. Lin Y, Chen D, Fan Q, Zhang H. Characterization of SoxB2 and SoxC genes in amphioxus (Branchiostoma belcheri): implications for their evolutionary conservation. Sci China C Life Sci. 2009;52:813-22.

16. Hinman VF, Nguyen AT, Cameron RA, Davidson EH. Developmental gene regulatory network architecture across 500 million years of echinoderm evolution. Proc Natl Acad Sci. 2003;100:13356-61.

17. Angerer $L M$, Newman $L A$, Angerer RC. SoxB1 downregulation in vegetal lineages of sea urchin embryos is achieved by both transcriptional repression and selective protein turnover. Development. 2005;132:999-1008.

18. Wei Z, Angerer RC, Angerer LM. Direct development of neurons within foregut endoderm of sea urchin embryos. Proc Natl Acad Sci USA. 2011:108:9143-7.

19. Garner S, Zysk I, Byrne G, Kramer M, Moller D, Taylor V, et al. Neurogenesis in sea urchin embryos and the diversity of deuterostome neurogenic mechanisms. Development. 2016:143:286-97.

20. Burke RD, Angerer LM, Elphick MR, Humphrey GW, Yaguchi S, Kiyama $\mathrm{T}$, et al. A genomic view of the sea urchin nervous system. Dev Biol. 2006;300:434-60

21. Burke RD, Osborne L, Wang D, Murabe N, Yaguchi S, Nakajima Y. Neuronspecific expression of a synaptotagmin gene in the sea urchin Strongylocentrotus purpuratus. J Comp Neurol. 2006:496:244-51.

22. Burke RD. Deuterostome neuroanatomy and the body plan paradox. Evol Dev. 2011;13:110-5.

23. Burke RD, Moller DJ, Krupke OA, Taylor VJ. Sea urchin neural development and the metazoan paradigm of neurogenesis. Genesis. 2014;52:208-21.

24. Barsi JC, Li E, Davidson EH. Geometric control of ciliated band regulatory states in the sea urchin embryo. Development. 2015;142:953-61.

25. Angerer LM, Yaguchi S, Angerer RC, Burke RD. The evolution of nervous system patterning: insights from sea urchin development. Development. 2011;138:3613-23.

26. Sun Z, Ettensohn CA. Signal-dependent regulation of the sea urchin skeletogenic gene regulatory network. Gene Expr Patterns. 2014;16:93-103.

27. Piacentino ML, Zuch DT, Fishman J, Rose S, Speranza EE, Li C, et al. RNASeq identifies SPGs as a ventral skeletal patterning cue in sea urchins. Development. 2016;143:703-14.
28. Kitajima T, Urakami H. Differential distribution of spicule matrix proteins in the sea urchin embryo skeleton. Dev Growth Differ. 2000;42:295-306.

29. Poustka AJ, Kühn A, Radosavljevic V, Wellenreuther R, Lehrach H, Panopoulou G. On the origin of the chordate central nervous system: expression of onecut in the sea urchin embryo. Evol Dev. 2004;6:227-36.

30. Richards GS, Rentzsch F. Regulation of Nematostella neural progenitors by SoxB, Notch and bHLH genes. Development. 2015;142:3332-42.

31. Richards GS, Rentzsch F. Transgenic analysis of a SoxB gene reveals neural progenitor cells in the cnidarian Nematostella vectensis. Development. 2014; $141: 4681-9$.

32. Meulemans D, Bronner-Fraser M. The amphioxus SoxB family: implications for the evolution of vertebrate placodes. Int J Biol Sci. 2007;3:356-64.

33. Ettensohn CA. Lessons from a gene regulatory network: echinoderm skeletogenesis provides insights into evolution, plasticity and morphogenesis. Development. 2009;136:11-21.

34. Koga $\mathrm{H}$, Morino $\mathrm{Y}$, Wada $\mathrm{H}$. The echinoderm larval skeleton as a possible model system for experimental evolutionary biology. Genesis. 2014:52:186-92.

35. Yu JKS, Holland LZ. Amphioxus whole-mount in situ hybridization. Cold Spring Harb. Protoc. 2009;2009:db.prot5286.

36. Cole AG, Arnone MI. Fluorescent in situ hybridization reveals multiple expression domains for SpBrn1/2/4 and identifies a unique ectodermal cell type that co-expresses the ParaHox gene SpLox. Gene Expr Patterns. 2009;9:324-8.

37. Nemer M, Rondinelli E, Infante D, Infante AA. Polyubiquitin RNA characteristics and conditional induction in sea urchin embryos. Dev Biol. 1991;145:255-65.

38. Hammond LM, Hofmann GE. Thermal tolerance of Strongylocentrotus purpuratus early life history stages: mortality, stress-induced gene expression and biogeographic patterns. Mar Biol. 2010;157:2677-87

\section{Submit your next manuscript to BioMed Central and we will help you at every step:}

- We accept pre-submission inquiries

- Our selector tool helps you to find the most relevant journal

- We provide round the clock customer support

- Convenient online submission

- Thorough peer review

- Inclusion in PubMed and all major indexing services

- Maximum visibility for your research

Submit your manuscript at www.biomedcentral.com/submit 